\title{
THE CHESS G-LINE WIGGLER TUNING *
}

\author{
Alexander B. Temnykh ${ }^{1)}$ and Kenneth D. Finkelstein ${ }^{2)}$ \\ 1) Laboratory of Nuclear Studies, ${ }^{2)}$ CHESS Cornell University, Ithaca NY 14853, USA
}

\begin{abstract}
The CHESS G-line wiggler has been tuned using a conventional magnetic measurement method together with a novel vibrating wire technique ${ }^{1}$. While the conventional method, based on magnetic field measurement with a long flip coil, provides magnetic field characteristics integrated over the wiggler length, the vibrating wire technique gives the location of magnetic field errors along the wiggler length allowing the correction of field errors in the location where they occurred.

The paper gives parameters of the CHESS G-line wiggler, describes the magnetic measurement setup, discusses some aspects of the vibrating wire technique applied to wiggler tuning, and reports on results of the CHESS G-line wiggler tune up.
\end{abstract}

\section{INTRODUCTION}

Cornell High Energy Synchrotron Source (CHESS) laboratory is based on the Cornell Electron Storage Ring (CESR) that also operates as electron-positron collider. At present two beam lines provide synchrotron radiation (SR) generated by permanent magnet wigglers. In the near future one of the present wigglers will be replaced by a recently built "G-line" wiggler. This wiggler has $12 \mathrm{~cm}$ period, composed of 50 permanent magnet poles of $11 \mathrm{~cm}$ width with $4 \mathrm{~cm}$ gap and $0.8 \mathrm{~T}$ of peak field.

Because CESR operates simultaneously as a synchrotron radiation source and a electron-positron collider, there are special requirements on wiggler multipole magnetic field errors. Table 2 shows maximum tolerable multipole magnetic field errors for CESR from reference [2], in comparison with errors evaluated for an APS ID from reference [3]. One can see that, while CESR's limits for dipole and quadrupole field errors $\left(b_{0}, a_{0}, b_{1}\right)$ are comparable or even larger than those at APS ${ }^{2}$, limits on skew quadrupole,

\footnotetext{
*mailto: abt6@cornell.edu, Work supported by National Since Foundation

${ }^{1}$ This technique is used to measure Fourier (sine) transform harmonics of the magnetic field distribution along the wire. Each harmonic is detected by the excitation of the corresponding mode of wire vibration. For this, an AC current with frequency in resonance with the frequency of the vibrating mode in question is driven through the wire. The vibration amplitude and phase (relative to the driving current) give the amplitude and sign of corresponding Fourier harmonics of the magnetic field distribution. Knowing harmonic one can reconstruct the field.

${ }^{2} a_{n}, b_{n}$ are coefficients of the magnetic field expansion: $\bar{B}_{y}+$ $i \bar{B}_{x}=\sum\left(a_{n}+i b_{n}\right)(x+i y)^{n} \cdot \bar{B}_{x, y}$ are magnetic field integrated over the wiggler length. First and second field integrals are: $I_{1 x, y}=$ $\int_{0}^{L_{w}} d z B_{x, y}(z), I_{2 x, y}=\int_{0}^{L_{w}} d z \int_{0}^{z} d z^{\prime} B_{x, y}\left(z^{\prime}\right), L_{w}$ is a wiggler
length.
}

\begin{tabular}{|c|c|c|c|}
\hline \hline Multipole coef. & CESR ID & APS ID & Units \\
\hline$b_{0}$ & 10.0 & 1.0 & $\mathrm{Gm}$ \\
$a_{0}$ & 10.0 & 1.0 & $\mathrm{Gm}$ \\
$b_{1}$ & 1.6 & 0.5 & $\mathrm{Gm} / \mathrm{cm}$ \\
$a_{1}$ & 0.23 & 0.5 & $\mathrm{Gm} / \mathrm{cm}$ \\
$b_{2}$ & 0.16 & 2.0 & $\mathrm{Gm} / \mathrm{cm}^{2}$ \\
$a_{2}$ & 0.02 & 0.1 & $\mathrm{Gm} / \mathrm{cm}^{2}$ \\
\hline$I_{2 x}$ & 17.6 & 10.0 & $\mathrm{Gm}{ }^{2}$ \\
$I_{2 y}$ & 17.6 & 10.0 & $\mathrm{Gm}{ }^{2}$ \\
\hline \hline
\end{tabular}

Table 1: Maximum tolerable field integrals and multipole errors integrated over the wiggler length.

sextupole and skew-sextupole components $\left(a_{1}, b_{2}, a_{2}\right)$ are several time smaller. One consequence of operating CESR with separated beams is the requirement of a large good field region.

The first objective in the wiggler tuning was to minimize first and second field integrals by tuning the end poles magnetic field strength. For this we used magnetic measurement based on the vibrating wire technique developed in [1]. Result was verified by a combination of Hall probe and long flip coil measurements. The second objective was correction of multipole field errors. It was found that the odd order normal multipole field errors such as $b_{1}, b_{3}$ and even order skew multipole errors like $a_{0}, a_{2}$ were due to misalignment of several wiggler poles in the horizontal plane, perpendicular to the beam axis. The vibrating wire technique localized regions along the wiggler where these errors occurred and allowed reduction of this effect by proper pole alignment. Others multipole components, $b_{2}$ and $a_{1}$, were corrected by putting $\mu$-metal shims on wiggler poles. The location for these shims was also indicated by vibrating wire measurements.

\section{MEASUREMENT SETUP}

Vibrating wire measurement setup was similar to that described elsewhere [1]. It consisted of copper-beryllium 100 micron diameter wire $396 \mathrm{~cm}$ in length, stretched through the wiggler. The wire mid point has been placed exactly in the middle of wiggler. The wire ends were fixed on stages movable in horizontal and vertical directions. At one end of the wire was a tension mechanism, while optical wire motion detectors were placed close to the opposite end. Wave form generator "HP33120A", controlled by a computer Macintosh Quadra 800, was used to drive AC current through the wire. A "LAB-NB" board installed in the Macintosh and programs based on "Lab-View" software pro- 
vided all needed signal analysis.

Long flip coil magnetic measurement setup based on techniques developed by the ID group at ESRF [4] consisted of a $\sim 4 \mathrm{~m}$ long 4 turns coil, (1), 3/8 inch width made with $1 / 64$ inch diameter copper wire stretched through the wiggler.

\section{FIRST AND SECOND FIELD INTEGRALS TUNING}

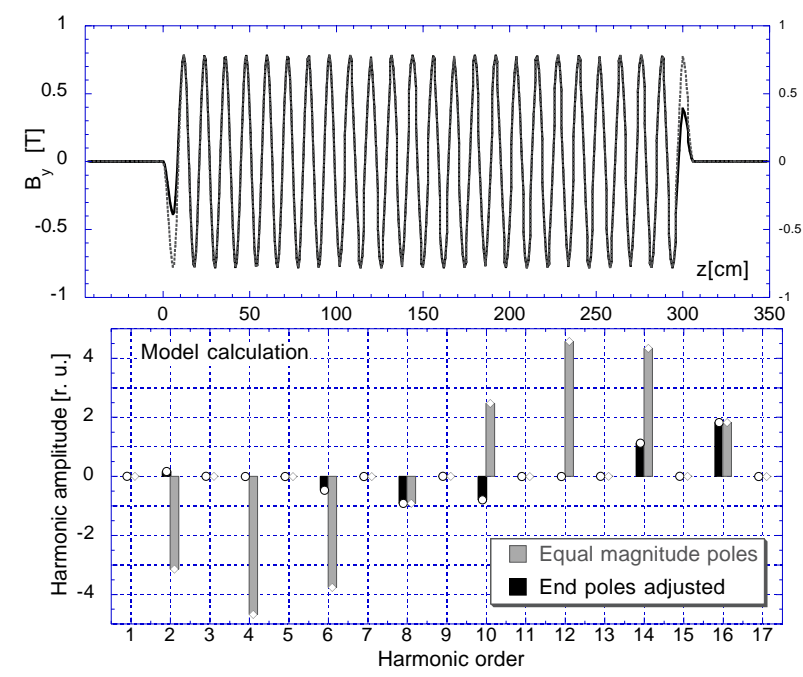

Figure 1: Model calculation. On upper plot shown vertical field as a function of coordinate along wiggler for 50 equal magnitude sinusoidal poles (dashed line) and for end poles strength adjusted to zero second field integral. On lower plot are the longitudinal harmonic amplitudes for equal magnitude poles (gray bars) and for adjusted (black bars).

The first and second integrals of the vertical magnetic field, $I_{1 y}, I_{2 y}$, see footnote 2 , cause the horizontal trajectory deflection and horizontal trajectory offset. Two poles on each end of CHESS G-line wiggler had mechanisms to enable field strength adjustment. Using the adjustments field integrals, $I_{1 y}$ and $I_{2 y}$, can be tuned to zero.

Figure 1 presents result of model calculation. Dashed line on upper plot shows wiggler field distribution along magnet for 50 uniform poles. Here $I_{1 y}=0$ and $I_{2 y}=$ $73.9 \mathrm{Gm}$ resulting in $-4.2 \mathrm{~mm}$ horizontal orbit offset. Solid line on the plot shows wiggler field when strength of end poles is adjusted to zero $I_{2 y}$. Here orbit offset is zero. Longitudinal harmonic of wiggler field, which can be measured directly with vibrating wire, see comments in footnote 1 , are depicted on lower plot. Here gray bars represent harmonics for uniform poles field distribution and black bars are for field distribution with adjusted end poles. One can see that end poles adjustment drastically reduced amplitudes of low order harmonics. Second and forth harmonics changed from -3.23 and -4.68 to 0.16 and -0.0067 r.u. This suggests a simple recipe for field integral tuning us- ing vibrating wire measurements; measure the first and the second Fourier harmonics with the fundamental and second wire vibrating modes while tuning end poles strength to minimize these values. Note that this recipe does work if regular poles have identical field strength.

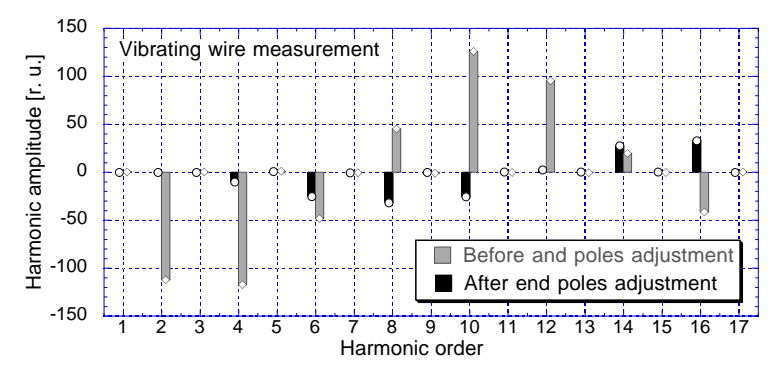

Figure 2: Vibrating wire measurement. Longitudinal harmonic of wiggler field measured before (dashed bars) and after (solid bars) end pole adjustment.

Figure 2 shows 17 lowes harmonics of the wiggler field measured before and after the end poles tuning. In the tuning process we measured first and second harmonics of the field distribution using the fundamental and second modes of wire vibration while adjusting the strength of the two end poles. One can see a good agreement seen between model calculation and measurement.

To verify results of the tuning we used a Hall probe to measure the strength of poles near both wiggler ends. Assuming identical regular poles in the middle of the wiggler, we estimated second field integral as $0.4 \mathrm{Gm}^{2}$. First field integral measured with a long flipping coil was $0.48 \mathrm{Gm}$. The results are well inside of specification given in table 2 .

\section{MULTIPOLE FIELD ERRORS CORRECTION}

Initial measurement with a long flipping coil stretched through the wiggler, shown in figure 3 and in table 2 revealed a multipole errors with a large $a_{2}$ (skew sextupole) component.

\begin{tabular}{|l|c|c|c|}
\hline \hline $\begin{array}{l}\text { Multi- } \\
\text { pole }\end{array}$ & Initial & $\begin{array}{c}\text { After poles } \\
\text { alignment }\end{array}$ & Final \\
\hline$b_{0}$ & $0.64 \pm 0.07$ & $-0.41 \pm 0.05$ & $0.0 \pm 0.07$ \\
$b_{1}$ & $0.54 \pm 0.04$ & $0.21 \pm 0.03$ & $0.32 \pm 0.04$ \\
$b_{2}$ & $0.21 \pm 0.01$ & $0.17 \pm 0.01$ & $0.0 \pm 0.01$ \\
$a_{0}$ & $-3.69 \pm 0.08$ & $-0.04 \pm 0.09$ & $-0.26 \pm 0.05$ \\
$a_{1}$ & $-0.86 \pm 0.04$ & $-0.92 \pm 0.05$ & $-0.03 \pm 0.02$ \\
$a_{2}$ & $0.63 \pm 0.02$ & $0.0 \pm 0.02$ & $-0.01 \pm 0.01$ \\
\hline \hline
\end{tabular}

Table 2: Multipole errors measured with long flip coil. Coefficients $a_{n}, b_{n}$ are given in $\mathrm{Gm} / \mathrm{cm}^{n}$.

Multipole error $a_{2}$ is resulted from a horizontal misalignment between top and bottom halves of wiggler poles in direction perpendicular to beam line. This component, $a_{2}$, can be easily corrected by the anti-parallel shift of any pole 


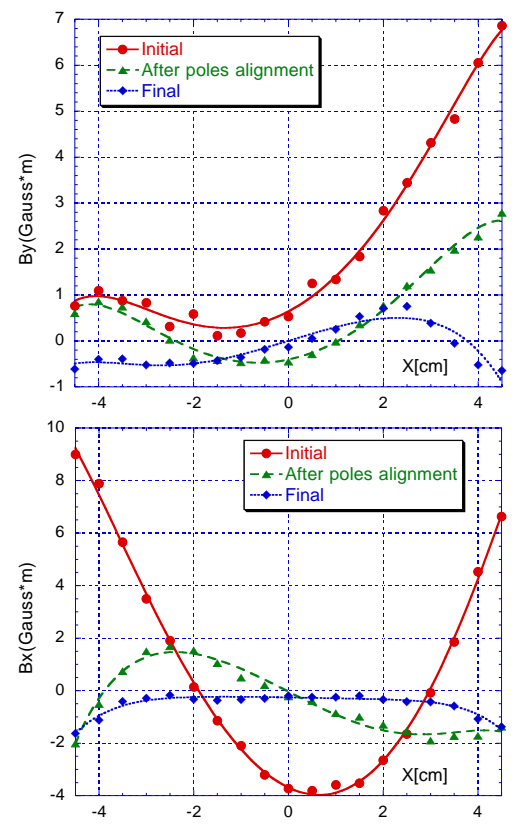

Figure 3: Results from flip coil measurements. Normal, $B_{y}$, and skew, $B_{x}$, integrated magnetic field errors measured with a long coil before tuning, after poles alignment, and after shims added to poles.

halves. However, from the beam dynamics point of view it is highly desired to correct multipole field errors in locations were they occur. This implies that magnetic field measurement techniques should provide information on the distribution of mutipole errors along the magnet. However, measurements with a long flipping coil do not locate field errors but instead provide the field integrated along the magnet. Another technique based on Hall probe scanning along the magnet requires a high precision setup and it is not clear the method can provide the required resolution when horizontal field errors are less than $10^{-3}$ of the strong vertical fields. These considerations motivated us to use the vibrating wire technique in the way described below.

We measured longitudinal harmonic of vertical and horizontal wiggler field at $x=-55,0,+55 \mathrm{~mm}$ of wire position using 3 lowest horizontal and vertical wire vibrating modes. Component $a_{2}$ is quadratic (symmetric) term in variation of horizontal field with $x$. Distribution of the symmetric component along magnet has been revealed in the following way. Using the measured harmonics we reconstructed horizontal field $B_{x}(x, z)$ at $x=-55,0,55 \mathrm{~mm}$ along magnet and then calculated symmetric part of the field variation: $\delta B_{x}^{s}(z)=B_{x}(x=-55, z)-2 B_{x}(x=$ $0, z)+B_{x}(x=55, z)$. Distribution of $\delta B_{x}^{s}$ along magnet, shown on figure 4 by solid line, indicated regions near $z \sim 90 \mathrm{~cm}$ and $z \sim 250 \mathrm{~cm}$ where top and bottom halves of poles are shifted relatively each other and direction of the shift. Few iterations of the measurement and poles alignment in the regions resulted in 6 times reduction of $\delta B^{s}$, see dashed line on 4 . Using measured harmonics of vertical field and doing similar analyzes, we revealed re-

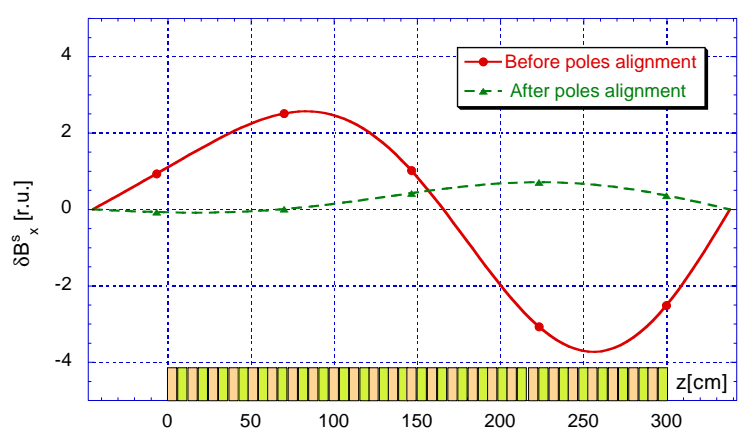

Figure 4: Results of vibrating wire measurement (3 harmonics ) applied to localizing field errors along the magnet. Symmetric part of horizontal field variation with $x$ shows distribution of even order skew multipoles ( skew sextupole like) along magnet before and after poles alignment (1r.u. $1 \mathrm{G)}$.

gions where the pole halves were shifted in the same direction and corrected this type of misalignment. Long flip coil measurement, made after the pole alignment, is plotted on figure 3 and is presented in table 2 . It indicated reduction of $b_{1}, b_{3}$, and elimination of skew-sextupole component $a_{2}$. Normal sextupole, $b_{2}$, and skew quadrupole, $a_{1}$, components were corrected by $20 \times 20 \mathrm{~mm}$ shims made with $\mu$-metal of $0.1 \mathrm{~mm}$ thickness. The appropriate location for shims along the magnet was also suggested from vibrating wire measurement. Plots on figure 3 and data in table 2 show results of final magnetic measurement. All field errors are below CESR tolerable limits shown in table 2 .

\section{CONCLUSION}

Field errors of CHESS G-line wiggler have been tuned to tolerable level by using two magnetic measurement techniques: the classical long flip coil measurement that gives field characteristics integrated over the wiggler length, and a novel vibrating wire technique. This second technique provides magnetic field characteristics along the magnet. It allowed us to tune second field integral to acceptable level as well as correct magnetic field multipole errors in locations where they occur.

\section{REFERENCES}

[1] A. Temnykh, Vibrating wire field-measuring technique, Nuc. Inst., A 399 (1997) 185-194

[2] Alexander Temnykh and David Rubin, On magnetic field quality of the East/West wiggler, Preprint CON 93-15.

[3] Glenn Decke, APS magnetioc specs, APS ID Acceptace Criteria based on Magnet Measurements by Efim Gluskin, Title II Design Review of Magnet Measurement Fa cility, APS, November 30, 1992.

[4] Developpement de Banc de measures magnetiques pour undulateurs et wigglers, D. Frachon Thesis, April 1992 